\title{
EXISTENCE OF POSITIVE SOLUTIONS FOR A SINGULAR FRACTIONAL NONLINEAR DIFFERENTIAL EQUATION WITH FRACTIONAL INTEGRAL BOUNDARY CONDITIONS
}

\author{
F. M. Gaafar \\ Faculty of Science, Damanhour University, Damanhour, Egypt \\ E-mail: fatmagaafar2@yahoo.com
}

Received 10/5/2018 Revised Accepted 21/5/2018

\begin{abstract}
In this paper, we investigate the appropriate conditions for the presence of positive solutions for a singular fractional differential equation (SFDE) with fractional integral boundary conditions (FIBCs). Our nonlinear function may has a singularity in its dependent variable. Our investigation is based on the fixed point theorem of Krasnosel'skii on a cone and via regularization and sequential techniques (RASTs).
\end{abstract}

Keywords and phrases. Fractional differential problem; positive solution; space singularity; integral boundary conditions; regularization; Sequential technique.

2010 Mathematics Subject Classification. 26A33, 34B16, 34B16.

\section{Introduction}

In this article, we establish the existence of at least one positive solution for the SFDE:

$$
D^{\alpha} u(t)+f\left(t, u(t), D^{\delta} u(t)\right)=0, \quad \alpha \in(1,2), \delta>0, \alpha-\delta \geq 1,
$$

with the FIBCs:

$$
u(1)=\lambda I^{\gamma} u(1),\left.\quad I^{\beta} u(t)\right|_{t=0}=0,
$$

where $D^{\alpha}$ is the Riemann-Liouville fractional derivative of order $\alpha, \gamma \in(0,1), \beta \in[0,2-\alpha), \lambda \geq 0, f$ is positive and satisfies the Carathéodory conditions on $[0,1] \times(0, \infty) \times R$ and $f(t, u, v)$ may be become singular at $u=0$.

Here the function $f:[0,1] \times(0, \infty) \times R$ satisfies the local Carathéodory conditions on the set $[0,1] \times \mathcal{M}, \mathcal{M}=(0, \infty) \times R$ $(f \in \operatorname{Car}([0,1] \times \mathcal{M}))$, if $f(., u, v)$ is measurable in $t \forall(u, v) \in(0, \infty) \times R$ and $f(t, . .$.$) is continuous in (u, v)$ for a.e. $t \in[0,1]$ and further for each compact set $\mathcal{K} \subset \mathcal{M}=(0, \infty) \times R$ there exists $\phi_{\mathcal{K}} \in L^{1}[0, \infty)$ such that

$$
|f(t, u, v)| \leq \phi_{\mathcal{K}}(t), \text { for a.e. } t \in[0,1], \forall(u, v) \in \mathcal{K} .
$$

The positive solutions for boundary value problems (BVPs) have received considerable attention owing to their importance in the mathematic theory and application (For details and examples, see Ahmad and Ntouyas [1], Bai [2], Baleanu et al. [3], Cabada, and Wang [4], El-Sayed et al. [5,6], Sabatier [7], Wei et al. [8], Zhao et al. [9], and the reference therein).

The investigation of singular BVPs for ordinary differential problems is moderately new, Indeed it was just in the center 1970s that scientists understood that large numbers of applications in the investigation of nonlinear phenomena gave rise 
to singular BVPs, however, as we would like to think, it was the 1979 Taliafcrro paper [10] that generated the interest of numerous researchers in singular problems in the 1980's and 1990's.

Singular boundary value problems (SBVPs) arise frequently in the study of nonlinear phenomena, for instance in nonNewtonian theory of fluid [10], Permeable Clarendon [11], appear in boundary layer theory [12], Electrostatic and gravitational forces. During the last few decades, the study of the existence of positive solutions for SBVPs has deserved the consideration of numerous researchers: with time singularities have been discussed by: Qui and Bai [13], Bai and Qui [14], Tian and Chen [15] and the reference therein. However, there are papers researching singular fractional BVPs which having singularities in dependent variables (see Agarwal et al. [16], Bai and Sun [17], Lyons and Neugebauer [18], Qiao and Zhou [19], Staněk [20], $\mathrm{Xu}$ et al. [21], Yuan et al. [22]).

Much consideration has been focused around the investigation of integral boundary conditions (IBCs), which are associated in various fields, for example, populations dynamics, chemical engineering and underground water flow, for a detailed description for FDEs with IBCs, we refer to some recent papers (see Cabada and Hamdi [23], Li et al. [24], Nanware and Dhaigude [25], Sun and Zhao [26], Zhang et al. [27], Wang and Xie [28]).

In [16], Agarwal et al. investigate the positivity and multiple solutions of the SFDE:

$$
D^{\alpha} u(t)+f\left(t, u(t), D^{\mu} u(t)\right)=0, \quad \alpha \in(1,2), \quad \mu>0, \quad \alpha-\mu \geq 1
$$

with

$$
u(0)=u(1)=0
$$

where $f>0$ satisfies the Carathédory conditions on $[0,1] \times(0, \infty) \times R$ and singular at $x=0$. The same last SFDE is considered by Qiao and Zhou [19], yet rather than the previous boundary conditions, they utilize the boundary conditions, $x(0)=D^{\beta} x(1)=0$ with $\beta \in(0, \alpha-1), \mu \in(0, \alpha-1)$ under similar conditions on $f$.

This paper is orderly as follows. In Section 2, we provided several preliminaries. In Sections 3, we convert SFDE with FIBCs (1.1)-(1.2) to an equivalent integral form and afterward acquire the Green's function and demonstrate a several properties of it. Existence result for the SFDE with FIBCs (1.1)-(1.2) is demonstrate by RASTs. At first we define a sequence of auxiliary regular FDE to the SFDE (1.1) and prove that the sequence of the auxiliary regular FDEs with FIBCs (4.4), (1.2) has a sequence $\left\{u_{m}\right\}$ of positive solutions which is relative compact (Lemmas 4.2 and 4.3). At last, in Section 5 , we construct a convergent subsequence of $\left\{u_{n_{m}}\right\}$ in $C[0,1]$, and afterward the Lebesgue dominated convergence theorem demonstrates that its limit $u$ is a solution of the SFDE with IBC (1.1)-(1.2) (Theorem 5.1).

\section{Preliminaries}

Here we presence definitions, preliminary facts, notations which are utilized all through this paper.

Let $\|u\|=\max \{|u(t)|: t \in[0,1]\}$ be the norm in the space $C[0,1]$ and $\|u\|_{L^{1}}=\int_{0}^{1}|u(t)| d t$ be the norm in $L^{1}[0,1]$, while $\|u\|_{0}=\max \left\{\|u\|,\left\|D^{\delta} u\right\|\right\}$.

Definition 2.1. (see [29]) The Riemann-Liouville fractional integral of the function $u(t) \in L^{1}[0,1]$ is identified as

$$
I^{\beta} u(t)=\int_{0}^{t} \frac{(t-s)^{\beta-1}}{\Gamma(\beta)} u(s) d s,
$$

and the Riemann-Liouville fractional derivative of order $n-1<\beta \leq n$ for the function $u(t)$ is identified as

$$
D^{\beta} u(t)=\frac{d^{n}}{d t^{n}} I^{n-\beta} u(t)=\frac{d^{n}}{d t^{n}} \int_{0}^{t} \frac{(t-s)^{n-\beta-1}}{\Gamma(n-\beta)} u(s) d s .
$$

Theorem 2.1. (Krasnosel'skii fixed point theorem [30]). Let $\mathcal{E}$ be a Banach space, and $\mathcal{Q} \subset \mathcal{E}$ be a cone in $\mathcal{E}$. Let $\Omega_{1}, \Omega_{2}$ be bounded open balls of $\mathcal{E}$ centered at the origin with $\bar{\Omega}_{1} \subset \Omega_{2}$. Suppose that $P: \mathcal{Q} \cap\left(\bar{\Omega}_{2} \backslash \Omega_{1}\right) \rightarrow \mathcal{Q}$ is a complete continuous operator such that either
(a) $\|P u\| \geq\|u\|$ for $u \in \mathcal{Q} \cap \partial \Omega_{1}$,
and $\quad\|P u\| \leq\|u\|$ for $u \in \mathcal{Q} \cap \partial \Omega_{2}$, or
(b) $\|P u\| \leq\|u\| \quad$ for $u \in \mathcal{Q} \cap \partial \Omega_{1}$,
and $\quad\|P u\| \geq\|u\|$ for $u \in \mathcal{Q} \cap \partial \Omega_{2}$

hold. Then $P$ has a fixed point in $\mathcal{Q} \cap\left(\bar{\Omega}_{2} \backslash \Omega_{1}\right)$. 
We list below assumptions to be used later in the paper.

$\left(H_{1}\right)$ The function $f \in \operatorname{Car}([0,1] \times \mathcal{M})$, for all $v \in R$, a.e. $t \in[0,1]$, and $\mathcal{M}=(0, \infty) \times R$

$$
\lim _{u \rightarrow 0^{+}} f(t, u, v)=\infty .
$$

$\left(H_{2}\right)$ for a.e. $t \in[0,1]$ and all $(u, v) \in \mathcal{M}$ there exists a constant $\rho>0$ such that .

$$
f(t, u, v) \geq \rho(1-t)^{2-2 \alpha}
$$

$\left(H_{3}\right) f$ satisfies the estimate

$$
f(t, u, v) \leq p_{0}(t)\left(p_{1}(t)+q(u)+r(u)+w(|v|)\right), \text { for a.e. } t \in[0,1], \text { and }(u, v) \in \mathcal{M},
$$

where $p_{0}, p_{1} \in L^{1}[0,1], q \in C(0, \infty)$ and non increasing function, $r, w \in C[0, \infty)$ are positive functions and nondecreasing,

$$
W=\int_{0}^{1} p_{0}(t) q\left(\frac{\rho t(1-t)^{2}}{2 \Gamma(\alpha)}\right) d t<\infty
$$

and

$$
\lim _{u \rightarrow \infty} \frac{r(u)+w(u)}{u}=0 .
$$

$\left(H_{4}\right) \quad \frac{\lambda \Gamma(\alpha)}{\Gamma(\alpha+\gamma)}<1$.

Remark 2.1. Under the assumption $\left(H_{1}\right)$, it follows that $\lim _{u \rightarrow 0^{+}} q(u)=\infty$.

\section{Green's function and its properties}

Lemma 3.1. [29] Let $\alpha \in(1,2]$. Then the following equality holds for $u \in L^{1}[0,1]$,

$$
I^{\alpha} D^{\alpha} u(t)=u(t)+c_{1} t^{\alpha-1}+c_{2} t^{\alpha-2}, \quad c_{i} \in R, i=1,2 .
$$

Lemma 3.2. Let $h(t) \in L^{1}[0,1]$, then the unique solution of the problem

$$
\begin{gathered}
D^{\alpha} u(t)+h(t)=0, \quad \alpha \in(1,2), \\
u(1)=\lambda I^{\gamma} u(1),\left.\quad I^{\beta} u(t)\right|_{t=0}=0,
\end{gathered}
$$

is obtained by

$$
u(t)=\int_{0}^{1} G(t, s) h(s) d s,
$$

where

$$
G(t, s)= \begin{cases}A t^{\alpha-1}\left[\frac{(1-s)^{\alpha-1}}{\Gamma(\alpha)}-\frac{\lambda(1-s)^{\alpha+\gamma-1}}{\Gamma(\alpha+\gamma)}\right]-\frac{(t-s)^{\alpha-1}}{\Gamma(\alpha)}, & 0 \leq s \leq t \leq 1 ; \\ A t^{\alpha-1}\left[\frac{(1-s)^{\alpha-1}}{\Gamma(\alpha)}-\frac{\lambda(1-s)^{\alpha+\gamma-1}}{\Gamma(\alpha+\gamma)}\right], & 0 \leq t \leq s \leq 1,\end{cases}
$$

and $A=\left(1-\frac{\lambda \Gamma(\alpha)}{\Gamma(\alpha+\gamma)}\right)^{-1}$.

Proof. We have the solution of (3.1) from Lemma 3.1 is given by

$$
\begin{aligned}
u(t) & =-I^{\alpha} h(t)+c_{1} t^{\alpha-1}+c_{2} t^{\alpha-2}, \\
I^{\beta} u(t) & =-I^{\alpha+\beta} h(t)+\frac{c_{1} \Gamma(\alpha)}{\Gamma(\alpha+\beta)} t^{\alpha+\beta-1}+\frac{c_{2} \Gamma(\alpha-1)}{\Gamma(\alpha+\beta-1)} t^{\alpha+\beta-2},
\end{aligned}
$$

and from $\left.I^{\beta} u(t)\right|_{t=0}=0$, we have $c_{2}=0$. Then

$$
u(t)=-I^{\alpha} h(t)+c_{1} t^{\alpha-1},
$$


and

$$
I^{\gamma} u(t)=-I^{\alpha+\gamma} h(t)+\frac{c_{1} \Gamma(\alpha)}{\Gamma(\alpha+\gamma)} t^{\alpha+\gamma-1}
$$

Therefore, we get

$$
I^{\gamma} u(1)=-\int_{0}^{1} \frac{(1-s)^{\alpha+\gamma-1}}{\Gamma(\alpha+\gamma)} h(s) d s+\frac{c_{1} \Gamma(\alpha)}{\Gamma(\alpha+\gamma)} .
$$

From $u(1)=\lambda I^{\gamma} u(1)$, we have

$$
c_{1}-\int_{0}^{1} \frac{(1-s)^{\alpha-1}}{\Gamma(\alpha)} h(s) d s=\frac{c_{1} \lambda \Gamma(\alpha)}{\Gamma(\alpha+\gamma)}-\lambda \int_{0}^{1} \frac{(1-s)^{\alpha+\gamma-1}}{\Gamma(\alpha+\gamma)} h(s) d s .
$$

Hence

$$
c_{1}=A\left[\int_{0}^{1} \frac{(1-s)^{\alpha-1}}{\Gamma(\alpha)} h(s) d s-\lambda \int_{0}^{1} \frac{(1-s)^{\alpha+\gamma-1}}{\Gamma(\alpha+\gamma)} h(s) d s\right] .
$$

We have that the solution of (3.1) can be expressed by the formula

$$
\begin{aligned}
u(t)= & A t^{\alpha-1}\left[\int_{0}^{1} \frac{(1-s)^{\alpha-1}}{\Gamma(\alpha)} h(s) d s-\lambda \int_{0}^{1} \frac{(1-s)^{\alpha+\gamma-1}}{\Gamma(\alpha+\gamma)} h(s) d s\right] \\
& -\int_{0}^{t} \frac{(t-s)^{\alpha-1}}{\Gamma(\alpha)} h(s) d s .
\end{aligned}
$$

Lemma 3.3. Assume that $\left(H_{4}\right)$ holds, then the function $G(t, s)$ defined by $(3.2)$ has the following properties:

(i) $G(t, s), t \in[0,1]$ is uniformly continuous;

(ii) $G(t, s) \geq 0 \forall(t, s) \in[0,1] \times[0,1]$;

(iii) $G(t, s) \leq \frac{A}{\Gamma(\alpha)}$ for all $(t, s) \in[0,1] \times[0,1]$.

Proof. It is easy to verify property (i), to show that (ii) holds.

Define $\quad g_{1}(t, s)=A t^{\alpha-1}\left[\frac{(1-s)^{\alpha-1}}{\Gamma(\alpha)}-\frac{\lambda(1-s)^{\alpha+\gamma-1}}{\Gamma(\alpha+\gamma)}\right]-\frac{(t-s)^{\alpha-1}}{\Gamma(\alpha)}$ for $0 \leq s \leq t \leq 1$, and

$$
g_{2}(t, s)=A t^{\alpha-1}\left[\frac{(1-s)^{\alpha-1}}{\Gamma(\alpha)}-\frac{\lambda(1-s)^{\alpha+\gamma-1}}{\Gamma(\alpha+\gamma)}\right] \text { for } 0 \leq t \leq s \leq 1 .
$$

Then for $0 \leq s \leq t \leq 1$, we have that

$$
\begin{aligned}
g_{1}(t, s) & =\frac{A t^{\alpha-1}}{\Gamma(\alpha)}\left[(1-s)^{\alpha-1}-\frac{\lambda \Gamma(\alpha)(1-s)^{\alpha+\gamma-1}}{\Gamma(\alpha+\gamma)}-\frac{1}{A}\left(1-\frac{s}{t}\right)^{\alpha-1}\right] \\
& \geq \frac{A t^{\alpha-1}(1-s)^{\alpha-1}}{\Gamma(\alpha)}\left[1-\frac{\lambda \Gamma(\alpha)(1-s)^{\gamma}}{\Gamma(\alpha+\gamma)}-\frac{1}{A}\right] \\
& \geq \frac{A t^{\alpha-1}(1-s)^{\alpha-1}}{\Gamma(\alpha)}\left[1-\frac{\lambda \Gamma(\alpha)}{\Gamma(\alpha+\gamma)}-\frac{1}{A}\right]=\frac{A t^{\alpha-1}(1-s)^{\alpha-1}}{\Gamma(\alpha)}\left[\frac{1}{A}-\frac{1}{A}\right]=0 .
\end{aligned}
$$

For $0 \leq t \leq s \leq 1$, we get that

$$
\begin{aligned}
g_{2}(t, s) & =A t^{\alpha-1}\left[\frac{(1-s)^{\alpha-1}}{\Gamma(\alpha)}-\frac{\lambda(1-s)^{\alpha+\gamma-1}}{\Gamma(\alpha+\gamma)}\right], \\
& \geq \frac{A t^{\alpha-1}(1-s)^{\alpha-1}}{\Gamma(\alpha)}\left[1-\frac{\lambda(1-s)^{\gamma} \Gamma(\alpha)}{\Gamma(\alpha+\gamma)}\right], \\
& \geq \frac{A t^{\alpha-1}(1-s)^{\alpha-1}}{\Gamma(\alpha)}\left[1-\frac{\lambda \Gamma(\alpha)}{\Gamma(\alpha+\gamma)}\right]=\frac{t^{\alpha-1}(1-s)^{\alpha-1}}{\Gamma(\alpha)} \geq 0 .
\end{aligned}
$$

Therefore, (ii) holds.

(iii) Since $\alpha>1,(t, s) \in[0,1] \times[0,1]$, then $t^{\alpha-1}$ and $(1-s)^{\alpha-1} \leq 1$. 
So from $(3.2), G(t, s) \leq \frac{A}{\Gamma(\alpha)}$ for $(t, s) \in[0,1] \times[0,1]$.

Lemma 3.4. Suppose that $h(t) \in L^{1}[0,1]$, let $h(t) \geq \rho(1-t)^{2-2 \alpha}$ for a.e. $t \in[0,1]$ with $\rho$ is a positive constant, then

$$
\int_{0}^{1} G(t, s) h(s) d s \geq \frac{\rho t(1-t)^{2}}{2 \Gamma(\alpha)} .
$$

Proof. Firstly, for $0 \leq s \leq t \leq 1$, using (3.4), we have

$$
\begin{aligned}
g_{1}(t, s) & \geq \frac{A t^{\alpha-1}(1-s)^{\alpha-1}}{\Gamma(\alpha)}\left[1-\frac{\lambda \Gamma(\alpha)(1-s)^{\gamma}}{\Gamma(\alpha+\gamma)}-\frac{1}{A}\right] \\
& \geq \frac{A t^{\alpha-1}(1-s)^{\alpha-1}}{\Gamma(\alpha)}\left[1-\frac{\lambda \Gamma(\alpha)(1-s)^{\gamma}}{\Gamma(\alpha+\gamma)}-1+\frac{\lambda \Gamma(\alpha)}{\Gamma(\alpha+\gamma)}\right] \\
& \geq \frac{A \lambda t^{\alpha-1}(1-s)^{\alpha-1}}{\Gamma(\alpha+\gamma)}\left[1-(1-s)^{\gamma}\right] \quad \text { (using Lagrange mean value theorem), } \\
& \geq \frac{A \lambda \gamma t^{\alpha-1}(1-s)^{\alpha-1} s}{\Gamma(\alpha+\gamma)} .
\end{aligned}
$$

Consequently, for $t \in[0,1]$ and $\gamma \in(0,1)$, and by using (3.5)

$$
\begin{aligned}
\int_{0}^{1} G(t, s) h(s) d s= & \int_{0}^{t} g_{1}(t, s) h(s) d s+\int_{t}^{1} g_{2}(t, s) h(s) d s \\
\geq & \int_{0}^{t} \frac{A \rho \lambda \gamma t^{\alpha-1}(1-s)^{\alpha-1}(1-s)^{2-2 \alpha} s}{\Gamma(\alpha+\gamma)} \\
& +\rho \int_{t}^{1} \frac{t^{\alpha-1}(1-s)^{\alpha-1}(1-s)^{2-2 \alpha}}{\Gamma(\alpha)} d s \\
\geq & \frac{A \rho \lambda \gamma t^{\alpha-1}}{\Gamma(\alpha+\gamma)} \int_{0}^{t}(1-s)^{1-\alpha} s d s+\frac{\rho t^{\alpha-1}}{\Gamma(\alpha)} \int_{t}^{1}(1-s)^{1-\alpha} d s \\
\geq & \frac{A \rho \lambda \gamma t^{\alpha-1}}{\Gamma(\alpha+\gamma)} \int_{0}^{t}(1-s) s d s+\frac{\rho t^{\alpha-1}}{\Gamma(\alpha)} \int_{t}^{1}(1-s) d s \\
\geq & \frac{\rho t^{\alpha-1}(1-t)^{2}}{2 \Gamma(\alpha)} \geq \frac{\rho t(1-t)^{2}}{2 \Gamma(\alpha)}, \quad t^{\alpha-1} \geq t, \text { for } t \in[0,1] .
\end{aligned}
$$

Define an operator $P_{m}$ by the formula

$$
\begin{aligned}
\left(P_{m} u\right)(t)= & \int_{0}^{1} G(t, s) f_{m}\left(s, u(s), D^{\delta} u(s)\right) d s, \quad 0 \leq t \leq 1 \\
= & A t^{\alpha-1}\left[\int_{0}^{1} \frac{(1-s)^{\alpha-1}}{\Gamma(\alpha)} f_{m}\left(s, u(s), D^{\delta} u(s)\right) d s\right. \\
& \left.-\lambda \int_{0}^{1} \frac{(1-s)^{\alpha+\gamma-1}}{\Gamma(\alpha+\gamma)} f_{m}\left(s, u(s), D^{\delta} u(s)\right) d s\right]-\int_{0}^{t} \frac{(t-s)^{\alpha-1}}{\Gamma(\alpha)} f_{m}\left(s, u(s), D^{\delta} u(s)\right) d s .
\end{aligned}
$$

Lemma 3.5. Suppose that $\left(H_{4}\right)$ holds, then we have

$$
\begin{aligned}
\left(D^{\delta} P_{m} u\right)(t)= & \frac{A \Gamma(\alpha) t^{\alpha-\delta-1}}{\Gamma(\alpha-\delta)}\left[\int_{0}^{1} \frac{(1-s)^{\alpha-1}}{\Gamma(\alpha)} f_{m}\left(s, u(s), D^{\delta} u(s)\right) d s\right. \\
& \left.-\lambda \int_{0}^{1} \frac{(1-s)^{\alpha+\gamma-1}}{\Gamma(\alpha+\gamma)} f_{m}\left(s, u(s), D^{\delta} u(s)\right) d s\right] \\
& -\int_{0}^{t} \frac{(t-s)^{\alpha-\delta-1}}{\Gamma(\alpha-\delta)} f_{m}\left(s, u(s), D^{\delta} u(s)\right) d s, \\
= & \int_{0}^{1} G^{*}(t, s) f_{m}\left(s, u(s), D^{\delta} u(s)\right) d s \in C[0,1], \quad 0 \leq t \leq 1 .
\end{aligned}
$$


Where $G^{*}(t, s)$ is the Green function defined by:

$$
G^{*}(t, s)= \begin{cases}\frac{A \Gamma(\alpha) t^{\alpha-\delta-1}}{\Gamma(\alpha-\delta)}\left[\frac{(1-s)^{\alpha-1}}{\Gamma(\alpha)}-\frac{\lambda(1-s)^{\alpha+\gamma-1}}{\Gamma(\alpha+\gamma)}\right]-\frac{(t-s)^{\alpha-\delta-1}}{\Gamma(\alpha-\delta)}, & 0 \leq s \leq t \leq 1 \\ \frac{A \Gamma(\alpha) t^{\alpha-\delta-1}}{\Gamma(\alpha-\delta)}\left[\frac{(1-s)^{\alpha-1}}{\Gamma(\alpha)}-\frac{\lambda(1-s)^{\alpha+\gamma-1}}{\Gamma(\alpha+\gamma)}\right], & 0 \leq t \leq s \leq 1\end{cases}
$$

and has the following properties:

(1) $G^{*}(t, s) \leq \frac{A}{\Gamma(\alpha-\delta)}$ for all $(t, s) \in[0,1] \times[0,1]$;

(2) $G^{*}(t, s) \in C([0,1] \times[0,1])$.

Proof. From Definition 2.1 and by using equation (3.6), let $h(r)=f_{m}\left(r, u(r), D^{\delta} u(r)\right)$ we have

$$
\begin{aligned}
& \left(D^{\delta} P_{m} u\right)(t)=\frac{d}{d t} \int_{0}^{t} \frac{(t-s)^{-\delta}}{\Gamma(1-\delta)}\left(P_{m} u\right)(s) d s, \\
& =\frac{d}{d t} \int_{0}^{t} \frac{(t-s)^{-\delta}}{\Gamma(1-\delta)}\left(\int_{0}^{1} G(s, r) h(r) d r\right) d s, \\
& =\frac{d}{d t} \int_{0}^{t} \frac{(t-s)^{-\delta}}{\Gamma(1-\delta)}\left[A s ^ { \alpha - 1 } \left(\int_{0}^{1} \frac{(1-r)^{\alpha-1}}{\Gamma(\alpha)} h(r) d r\right.\right. \\
& \left.\left.-\lambda \int_{0}^{1} \frac{(1-r)^{\alpha+\gamma-1}}{\Gamma(\alpha+\gamma)} h(r) d r\right)-\int_{0}^{s} \frac{(s-r)^{\alpha-1}}{\Gamma(\alpha)} h(r) d r\right] d s, \\
& =A \frac{d}{d t} \int_{0}^{t} \frac{(t-s)^{-\delta} s^{\alpha-1}}{\Gamma(1-\delta)} d s\left(\int_{0}^{1} \frac{(1-r)^{\alpha-1}}{\Gamma(\alpha)} h(r) d r\right. \\
& \left.-\lambda \int_{0}^{1} \frac{(1-r)^{\alpha+\gamma-1}}{\Gamma(\alpha+\gamma)} h(r) d r\right)-\frac{d}{d t} \int_{0}^{t} \frac{(t-s)^{-\delta}}{\Gamma(1-\delta)} d s \int_{0}^{s} \frac{(s-r)^{\alpha-1}}{\Gamma(\alpha)} h(r) d r, \\
& =\frac{d}{d t} \frac{A \Gamma(\alpha) t^{\alpha-\delta}}{\Gamma(1+\alpha-\delta)}\left[\int_{0}^{1} \frac{(1-r)^{\alpha-1}}{\Gamma(\alpha)} h(r) d r-\lambda \int_{0}^{1} \frac{(1-r)^{\alpha+\gamma-1}}{\Gamma(\alpha+\gamma)} h(r) d r\right] \\
& -\frac{d}{d t} \int_{0}^{t} \frac{(t-s)^{\alpha-\delta}}{\Gamma(1+\alpha-\delta)} h(s) d s, \\
& =\frac{A \Gamma(\alpha) t^{\alpha-\delta-1}}{\Gamma(\alpha-\delta)}\left[\int_{0}^{1} \frac{(1-s)^{\alpha-1}}{\Gamma(\alpha)} h(s) d s-\lambda \int_{0}^{1} \frac{(1-s)^{\alpha+\gamma-1}}{\Gamma(\alpha+\gamma)} h(s) d s\right] \\
& -\int_{0}^{t} \frac{(t-s)^{\alpha-\delta-1}}{\Gamma(\alpha-\delta)} h(s) d s, \\
& =\int_{0}^{1} G^{*}(t, s) h(s) d s, \quad 0 \leq t \leq 1 .
\end{aligned}
$$

We now show that (1) and (2) are true.

(1) It is easy to prove $G^{*}(t, s) \leq \frac{A}{\Gamma(\alpha-\delta)}$ for all $(t, s) \in[0,1] \times[0,1]$.

(2) Since $\alpha-\delta \geq 1$. Hence $G^{*}(t, s) \in C([0,1] \times[0,1])$ and $D^{\delta} P_{m} u \in C[0,1]$ (notice that $\int_{0}^{t} \frac{(t-s)^{\alpha-\delta-1}}{\Gamma(\alpha-\delta)} h(s) d s \in C[0,1]$ ).

\section{Auxiliary regular FDE}

If assumption $\left(H_{1}\right)$ is satisfied, then equation (1.1) is singular equation and we apply RASTs for the existence of positive solutions of (1.1)-(1.2).

Let $\mathcal{E}=\left\{u \in C[0,1]: D^{\delta} u \in C[0,1]\right\}$ endowed with the norm $\|u\|_{0}=\max \left\{\|u\|,\left\|D^{\delta} u\right\|\right\}$, then $\mathcal{E}$ is a Banach space (see [31]). Let $\mathcal{Q}=\{u \in \mathcal{E}: u(t) \geq 0$ for $t \in[0,1]\}$, then $\mathcal{Q}$ is a cone in $\mathcal{E}$.

Define the function $f_{m}, m \in N$, by the formula

$$
f_{m}(t, u, v)= \begin{cases}f(t, u, v) & \text { if } u \geq \frac{1}{m} \\ f\left(t, \frac{1}{m}, v\right) & \text { if } 0 \leq u<\frac{1}{m}\end{cases}
$$


Then $f_{m} \in \operatorname{Car}\left([0,1] \times \mathcal{M}^{*}\right), \mathcal{M}^{*}=[0, \infty) \times R$, and assumptions $\left(H_{2}\right)$ and $\left(H_{3}\right)$ give

$$
\begin{aligned}
f_{m}(t, u, v) \geq & \rho(1-t)^{2-2 \alpha} \quad \text { for a.e. } t \in[0,1] \text { and all }(u, v) \in \mathcal{M}^{*} . \\
f_{m}(t, u, v) \leq & p_{0}(t)\left(p_{1}(t)+q\left(\frac{1}{m}\right)+r(u)+r(1)+w(|v|)\right) \\
& \text { for a.e. } t \in[0,1] \text { and all }(u, v) \in \mathcal{M}^{*}, \text { and } \\
f_{m}(t, u, v) \leq & p_{0}(t)\left(p_{1}(t)+q(u)+r(u)+r(1)+w(|v|)\right) \\
& \text { for a.e. } t \in[0,1] \text { and all }(u, v) \in \mathcal{M}=(0, \infty) \times R .
\end{aligned}
$$

Consider the family of regular differential equations

$$
D^{\alpha} u(t)+f_{m}\left(t, u(t), D^{\delta} u(t)\right)=0, \quad \alpha \in(1,2), 0<\delta<1, \alpha-\delta \geq 1,
$$

with fractional integral boundary condition (1.2).

Definition 4.1. A function $u \in C[0,1]$ is a positive solution to (4.4), (1.2) if $u>0$ on $[0,1], D^{\delta} u(t) \in C[0,1], D^{\alpha} u(t) \in$ $L^{1}[0,1]$ and $(4.4),(1.2)$ hold a.e. on $[0,1]$.

Lemma 4.1. Suppose that $\left(H_{1}\right)-\left(H_{4}\right)$ hold. Then $P_{m}: \mathcal{Q} \rightarrow \mathcal{Q}$ and $P_{m}$ is a completely continuous operator.

Proof. Let $u \in \mathcal{Q}$ and let $h(t)=f_{m}\left(t, u(t), D^{\delta} u(t)\right)$ for a.e. $0 \leq t \leq 1$. Then $h(t) \geq 0$ and $h(t) \in L^{1}[0,1]$. In order to prove that $P_{m}: \mathcal{Q} \rightarrow \mathcal{Q}$, we have the equality (cf. (3.6))

$$
\begin{aligned}
\left(P_{m} u\right)(t)= & \int_{0}^{1} G(t, s) h(s) d s \\
= & A t^{\alpha-1}\left[\int_{0}^{1} \frac{(1-s)^{\alpha-1}}{\Gamma(\alpha)} h(s) d s-\lambda \int_{0}^{1} \frac{(1-s)^{\alpha+\gamma-1}}{\Gamma(\alpha+\gamma)} h(s) d s\right] \\
& -\int_{0}^{t} \frac{(t-s)^{\alpha-1}}{\Gamma(\alpha)} h(s) d s .
\end{aligned}
$$

Notice from $\int_{0}^{t} \frac{(t-s)^{\alpha-1}}{\Gamma(\alpha)} h(s) d s \in C[0,1]$ and from $G \geq 0$ by Lemma 3.3(ii) that, for $u \in \mathcal{Q}$ we have $P_{m} u \in C[0,1]$ and $\left(P_{m} u\right)(t) \geq 0$ on $[0,1]$. Likewise, from Lemma 3.5 we have $\left(D^{\delta} P_{m} u\right)(t) \in C[0,1]$ for $t \in[0,1]$. Consequently, $P_{m}: \mathcal{Q} \rightarrow \mathcal{Q}$.

Secondly, we prove that $P_{m}$ is bounded on any bounded set from $\mathcal{Q}$. Let $\mathcal{K} \subset \mathcal{Q}$ ba a bounded subset in $\mathcal{Q}$. Since $f_{m} \in \operatorname{Car}\left([0,1] \times \mathcal{M}^{*}\right)$, there exists $\phi \in L^{1}[0,1]$ such that

$$
0<f_{m}\left(t, u(t), D^{\delta} u(t)\right) \leq \phi(t) \quad \forall u \in \mathcal{K} \text { and a.e. } t \in[0,1] .
$$

From Lemma 3.3, we have

$$
\begin{aligned}
\left|\left(P_{m} u\right)(t)\right| & =\left|\int_{0}^{1} G(t, s) f_{m}\left(s, u(s), D^{\delta} u(s)\right) d s\right| \\
& \leq \frac{A}{\Gamma(\alpha)} \int_{0}^{1} f_{m}\left(s, u(s), D^{\delta} u(s)\right) d s \leq \frac{A}{\Gamma(\alpha)}\|\phi\|_{L^{1}} .
\end{aligned}
$$

Also from Lemma 3.5, we obtain

$$
\left|\left(D^{\delta} P_{m} u\right)(t)\right|=\left|\int_{0}^{1} G^{*}(t, s) f_{m}\left(s, u(s), D^{\delta} u(s)\right) d s\right| \leq \frac{A}{\Gamma(\alpha-\delta)}\|\phi\|_{L^{1}} .
$$

Hence $P_{m}(\mathcal{K})$ is bounded in $\mathcal{Q}$.

Thirdly, we show that $P_{m}$ is a continuous operator. Let $\left\{u_{k}\right\} \subset \mathcal{Q}$ be a convergent sequence to $u$ in $\mathcal{Q}$, then $\lim _{k \rightarrow \infty} \| u_{k}-$ $u \|_{0}=0$ and then $u \in \mathcal{Q}$.

Also by Lemma 3.3(iii), we have

$$
\left|\left(P_{m} u_{k}\right)(t)-\left(P_{m} u\right)(t)\right| \leq \frac{A}{\Gamma(\alpha)} \int_{0}^{1}\left|f_{m}\left(s, u_{k}(s), D^{\delta} u_{k}(s)\right)-f_{m}\left(s, u(s), D^{\delta} u(s)\right)\right| d s .
$$


Since $f_{m} \in \operatorname{Car}\left([0,1] \times \mathcal{M}^{*}\right)$, then we have

$$
\lim _{k \rightarrow \infty} f_{m}\left(t, u_{k}(t), D^{\delta} u_{k}(t)\right)=f_{m}\left(t, u(t), D^{\delta} u(t)\right) .
$$

By applying Lebesgue dominated convergence theorem and using (4.2) we obtain,

$$
\lim _{k \rightarrow \infty} \int_{0}^{1}\left|f_{m}\left(t, u_{k}(t), D^{\delta} u_{k}(t)\right)-f_{m}\left(t, u(t), D^{\delta} u(t)\right)\right|=0 .
$$

Hence we get

$$
\left|\left(P_{m} u_{k}\right)(t)-\left(P_{m} u\right)(t)\right| \rightarrow 0 \quad \text { as } \quad k \rightarrow \infty .
$$

Also from (3.7) we have

$$
\begin{aligned}
\leq & \frac{A \Gamma(\alpha) t^{\alpha-\delta-1}}{\Gamma(\alpha-\delta)} \int_{0}^{1} \frac{(1-s)^{\alpha-1}}{\Gamma(\alpha)}\left|f_{m}\left(s, u_{k}(s), D^{\delta} u_{k}(s)\right)-f_{m}\left(s, u(s), D^{\delta} u(s)\right)\right| d s \\
& +\frac{A \lambda \Gamma(\alpha) t^{\alpha-\delta-1}}{\Gamma(\alpha-\delta)} \int_{0}^{1} \frac{(1-s)^{\alpha+\gamma-1}}{\Gamma(\alpha+\gamma)}\left|f_{m}\left(s, u_{k}(s), D^{\delta} u_{k}(s)\right)-f_{m}\left(s, u(s), D^{\delta} u(s)\right)\right| d s \\
& +\int_{0}^{t} \frac{(t-s)^{\alpha-\delta-1}}{\Gamma(\alpha-\delta)}\left|f_{m}\left(s, u_{k}(s), D^{\delta} u_{k}(s)\right)-f_{m}\left(s, u(s), D^{\delta} u(s)\right)\right| d s, \\
\leq & \frac{A}{\Gamma(\alpha-\delta)} \int_{0}^{1}\left|f_{m}\left(s, u_{k}(s), D^{\delta} u_{k}(s)\right)-f_{m}\left(s, u(s), D^{\delta} u(s)\right)\right| d s \\
& +\frac{A \lambda \Gamma(\alpha)}{\Gamma(\alpha-\delta) \Gamma(\alpha+\gamma)} \int_{0}^{1}\left|f_{m}\left(s, u_{k}(s), D^{\delta} u_{k}(s)\right)-f_{m}\left(s, u(s), D^{\delta} u(s)\right)\right| d s \\
& +\frac{1}{\Gamma(\alpha-\delta)} \int_{0}^{1}\left|f_{m}\left(s, u_{k}(s), D^{\delta} u_{k}(s)\right)-f_{m}\left(s, u(s), D^{\delta} u(s)\right)\right| d s, \\
& \rightarrow 0 \text { as } \quad k \rightarrow \infty .
\end{aligned}
$$

Therefor $\lim _{m \rightarrow \infty}\left\|\left(P_{m} u_{k}\right)(t)-\left(P_{m} u\right)(t)\right\|_{0}=0$, that is $P_{m}$ is a continuous operator.

Finally, to prove $P_{m}$ is an equicontinuous operator. Let $0 \leq t_{1}<t_{2} \leq 1$, then using (4.5) we obtain

$$
\begin{aligned}
\left|\left(P_{m} u\right)\left(t_{2}\right)-\left(P_{m} u\right)\left(t_{1}\right)\right| \leq & A\left(t_{2}^{\alpha-1}-t_{1}^{\alpha-1}\right)\left[\int_{0}^{1} \frac{(1-s)^{\alpha-1}}{\Gamma(\alpha)} f_{m}\left(s, u(s), D^{\delta} u(s)\right) d s\right. \\
& \left.+\lambda \int_{0}^{1} \frac{(1-s)^{\alpha+\gamma-1}}{\Gamma(\alpha+\gamma)} f_{m}\left(s, u(s), D^{\delta} u(s)\right) d s\right] \\
& +\int_{0}^{t_{1}} \frac{\left(t_{2}-s\right)^{\alpha-1}-\left(t_{1}-s\right)^{\alpha-1}}{\Gamma(\alpha)} f_{m}\left(s, u(s), D^{\delta} u(s)\right) d s \\
& +\int_{t_{1}}^{t_{2}} \frac{\left(t_{2}-s\right)^{\alpha-1}}{\Gamma(\alpha)} f_{m}\left(s, u(s), D^{\delta} u(s)\right) d s, \\
\leq & \frac{A}{\Gamma(\alpha)}\left(1+\frac{\lambda \Gamma(\alpha)}{\Gamma(\alpha+\gamma)}\right)\left(t_{2}^{\alpha-1}-t_{1}^{\alpha-1}\right)\|\phi\|_{L^{1}} \\
& +\frac{\left(t_{2}-t_{1}\right)^{\alpha-1}}{\Gamma(\alpha)} \int_{t_{1}}^{t_{2}} \phi(s) d s+\int_{0}^{t_{1}} \frac{\left(t_{2}-s\right)^{\alpha-1}-\left(t_{1}-s\right)^{\alpha-1}}{\Gamma(\alpha)} \phi(s) d s .
\end{aligned}
$$


Also from (3.7) we have

$$
\begin{aligned}
\left|\left(D^{\delta} P_{m} u\right)\left(t_{2}\right)-\left(D^{\delta} P_{m} u\right)\left(t_{1}\right)\right| & \\
\leq & \frac{A \Gamma(\alpha)\left(t_{2}^{\alpha-\delta-1}-t_{1}^{\alpha-\delta-1}\right)}{\Gamma(\alpha-\delta)}\left[\int_{0}^{1} \frac{(1-s)^{\alpha-1}}{\Gamma(\alpha)} f_{m}\left(s, u(s), D^{\delta} u(s)\right) d s\right. \\
& \left.+\lambda \int_{0}^{1} \frac{(1-s)^{\alpha+\gamma-1}}{\Gamma(\alpha+\gamma)} f_{m}\left(s, u(s), D^{\delta} u(s)\right) d s\right] \\
& +\int_{0}^{t_{1}} \frac{\left(t_{2}-s\right)^{\alpha-\delta-1}-\left(t_{1}-s\right)^{\alpha-\delta-1}}{\Gamma(\alpha-\delta)} f_{m}\left(s, u(s), D^{\delta} u(s)\right) d s \\
& +\int_{t_{1}}^{t_{2}} \frac{\left(t_{2}-s\right)^{\alpha-\delta-1}}{\Gamma(\alpha-\delta)} f_{m}\left(s, u(s), D^{\delta} u(s)\right) d s, \\
\leq & \frac{A\left(t_{2}^{\alpha-\delta-1}-t_{1}^{\alpha-\delta-1}\right)}{\Gamma(\alpha-\delta)}\left(1+\frac{\lambda \Gamma(\alpha)}{\Gamma(\alpha+\gamma)}\right)\|\phi\|_{L^{1}} \\
& +\int_{0}^{t_{1}} \frac{\left(t_{2}-s\right)^{\alpha-\delta-1}-\left(t_{1}-s\right)^{\alpha-\delta-1}}{\Gamma(\alpha-\delta)} \phi(s) d s+\frac{\left(t_{2}-t_{1}\right)^{\alpha-\delta-1}}{\Gamma(\alpha-\delta)} \int_{t_{1}}^{t_{2}} \phi(s) d s, \quad \text { if } \alpha-\delta>1,
\end{aligned}
$$

and

$$
\left|\left(D^{\delta} P_{m} u\right)\left(t_{2}\right)-\left(D^{\delta} P_{m} u\right)\left(t_{1}\right)\right| \leq \int_{t_{1}}^{t_{2}} \phi(s) d s, \quad \text { if } \alpha-\delta=1 .
$$

As $t_{2} \rightarrow t_{1}$, the right side of the above inequalities tend to zero. Therefore $\left\{P_{m} u: u \in \mathcal{K}\right\}$ and $\left\{D^{\delta} P_{m} u: u \in \mathcal{K}\right\}$ are bounded in $C[0,1]$ and equicontinuous on $[0,1]$, and by the Arzelá-Ascoli theorem $P_{m}(\mathcal{K})$ is relative compact and we have $P_{m}$ is a completely continuous operator.

We now apply Krasnosel'skii fixed point theorem to determine that $P_{m}$ has a fixed point theorem in $\mathcal{Q} \cap\left(\overline{\Omega_{2}} \backslash \Omega_{1}\right)$. Accordingly, problem (4.4), (1.2) has a multiple solutions.

Lemma 4.2. Assume that $\left(H_{1}\right)-\left(H_{4}\right)$ hold. Then the auxiliary regular FDE (4.4) with FIBCs (1.2) has multiple positive solutions.

Proof. By Lemma $4.1 P_{m}: \mathcal{Q} \rightarrow \mathcal{Q}$ is completely continuous operator.

Define $\Omega_{1}=\left\{u \in \mathcal{E}:\|u\|_{0}<\frac{2 \rho}{27 \Gamma(\alpha)}\right\}$.

Since $\left\|P_{m} u\right\|_{0}=\max \left\{\left\|P_{m} u\right\|,\left\|D^{\delta} P_{m} u\right\|\right\}$, hence $\left\|P_{m} u\right\|_{0} \geq\left\|P_{m} u\right\|=\left\{\max \left|P_{m} u(t)\right|: t \in[0,1]\right\}$.

From Lemma $3.4\left(P_{m} u\right)(t) \geq \frac{\rho t(1-t)^{2}}{2 \Gamma(\alpha)}, u \in \mathcal{Q}$ and we have $\left\|P_{m} u\right\|_{0} \geq \max _{t \in[0,1]} \frac{\rho t(1-t)^{2}}{2 \Gamma(\alpha)}$. Hence $\left\|P_{m} u\right\|_{0} \geq \frac{2 \rho}{27 \Gamma(\alpha)}$ and therefore

$$
\left\|P_{m} u\right\|_{0} \geq\|u\|_{0} \text { for } u \in \mathcal{Q} \cap \partial \Omega_{1} .
$$

Next, from inequality (4.2) and Lemma 3.3(iii)

$$
\begin{aligned}
\left|\left(P_{m} u\right)(t)\right| & \leq \frac{A}{\Gamma(\alpha)} \int_{0}^{1} p_{0}(s)\left(p_{1}(s)+q\left(\frac{1}{m}\right)+r(u(s))+r(1)+w\left(\left|D^{\delta} u(s)\right|\right)\right) d s, \\
& \leq \frac{A}{\Gamma(\alpha)} \int_{0}^{1} p_{0}(s)\left(p_{1}(s)+q\left(\frac{1}{m}\right)+r(\|u\|)+r(1)+w\left(\left\|D^{\delta} u\right\|\right)\right) d s, \\
& \leq \frac{A}{\Gamma(\alpha)}\left[\left\|p_{0} p_{1}\right\|_{L^{1}}+\left(q\left(\frac{1}{m}\right)+r(\|u\|)+r(1)+w\left(\left\|D^{\delta} u\right\|\right)\right)\left\|p_{0}\right\|_{L^{1}}\right] .
\end{aligned}
$$

Also from Lemma 3.5 and inequality (4.2), we have

$$
\left|\left(D^{\delta} P_{m} u\right)(t)\right| \leq \frac{A}{\Gamma(\alpha-\delta)}\left[\left\|p_{0} p_{1}\right\|_{L^{1}}+\left(q\left(\frac{1}{m}\right)+r(\|u\|)+r(1)+w\left(\left\|D^{\delta} u\right\|\right)\right)\left\|p_{0}\right\|_{L^{1}}\right],
$$

because $r, w$ are nondecreasing by $\left(H_{3}\right)$. Let $\Upsilon=\max \left\{\frac{A}{\Gamma(\alpha)}, \frac{A}{\Gamma(\alpha-\delta)}\right\}$. Hence for $u \in \mathcal{E}$, we have

$$
\left\|P_{m} u\right\|_{0} \leq \Upsilon\left[\left\|p_{0} p_{1}\right\|_{L^{1}}+\left(q\left(\frac{1}{m}\right)+r\left(\|u\|_{0}\right)+r(1)+w\left(\|u\|_{0}\right)\right)\left\|p_{0}\right\|_{L^{1}}\right] .
$$


Now from condition $\left(H_{3}\right)$, since $\lim _{\vartheta \rightarrow \infty} \frac{r(\vartheta)+w(\vartheta)}{\vartheta}=0$, then there exists an adequately large number $S>0$ such that

$$
\Upsilon\left[\left\|p_{0} p_{1}\right\|_{L^{1}}+\left(q\left(\frac{1}{m}\right)+r(S)+r(1)+w(S)\right)\left\|p_{0}\right\|_{L^{1}}\right]<S .
$$

Let $\Omega_{2}=\left\{u \in \mathcal{E}:\|u\|_{0}<S\right\}$. Then for $u \in \mathcal{Q} \cap \partial \Omega_{2}$ and $t \in[0,1]$, it follows from the last two inequalities that,

$$
\left\|P_{m} u\right\|_{0} \leq\|u\|_{0} \text { for } u \in \mathcal{Q} \cap \partial \Omega_{2} .
$$

Applying Theorem 2.1, we deduce from (4.6) and (4.7) that $P_{m}$ has a fixed point in $\mathcal{Q} \cap\left(\overline{\Omega_{2}} \backslash \Omega_{1}\right)$, thus auxiliary regular FDE (4.4) with FIBCs (1.2) has multiple positive solutions.

Now, we deal with a sequence of solutions of regular FDE (4.4) with FIBCs (1.2).

Lemma 4.3. Suppose that $\left(H_{1}\right)-\left(H_{4}\right)$ hold. Let $u_{m}$ be a solution of $(4.4),(1.2)$ defined by

$$
u_{m}(t)=\int_{0}^{1} G(t, s) f_{m}\left(s, u_{m}(s), D^{\delta} u_{m}(s)\right) d s, m \in N, t \in[0,1]
$$

Then the sequences $\left\{u_{m}\right\}$ and $\left\{D^{\delta} u_{m}\right\}$ are relatively compact in $C[0,1]$.

Proof. We prove this lemma by a same route as the proof of Lemma 4.1 , for $t \in[0,1], m \in N$, we have (cf. (3.7))

$$
\begin{aligned}
D^{\delta} u_{m}(t)= & \frac{A \Gamma(\alpha) t^{\alpha-\delta-1}}{\Gamma(\alpha-\delta)}\left[\int_{0}^{1} \frac{(1-s)^{\alpha-1}}{\Gamma(\alpha)} f_{m}\left(s, u_{m}(s), D^{\delta} u_{m}(s)\right) d s\right. \\
& \left.-\lambda \int_{0}^{1} \frac{(1-s)^{\alpha+\gamma-1}}{\Gamma(\alpha+\gamma)} f_{m}\left(s, u_{m}(s), D^{\delta} u_{m}(s)\right) d s\right] \\
& -\int_{0}^{t} \frac{(t-s)^{\alpha-\delta-1}}{\Gamma(\alpha-\delta)} f_{m}\left(s, u_{m}(s), D^{\delta} u_{m}(s)\right) d s
\end{aligned}
$$

It follow from (4.1) and Lemma 3.4 that:

$$
u_{m}(t) \geq \frac{\rho t(1-t)^{2}}{2 \Gamma(\alpha)}
$$

but from (4.3) and $\left(H_{3}\right)$, we have

$$
\begin{aligned}
f_{m}\left(t, u_{m}, D^{\delta} u_{m}(t)\right) & \leq p_{0}(t)\left(p_{1}(t)+q\left(u_{m}(t)\right)+r\left(u_{m}(t)\right)+r(1)+w\left(\left|D^{\delta} u_{m}(t)\right|\right)\right), \\
& \leq p_{0}(t)\left(p_{1}(t)+q\left(\frac{\rho t(1-t)^{2}}{2 \Gamma(\alpha)}\right)+r\left(\left\|u_{m}\right\|_{0}\right)+r(1)+w\left(\left\|u_{m}\right\|_{0}\right) .\right.
\end{aligned}
$$

This implies (cf. (2.1), (4.8), (4.9) and (4.10))

$$
\begin{aligned}
\left|u_{m}(t)\right| \leq & \frac{A}{\Gamma(\alpha)}\left[\left\|p_{0} p_{1}\right\|_{L^{1}}+\int_{0}^{1} p_{0}(t) q\left(\frac{\rho t(1-t)^{2}}{2 \Gamma(\alpha)}\right) d t+\left(r\left(\left\|u_{m}\right\|_{0}\right)+r(1)\right.\right. \\
& \left.\left.+w\left(\left\|u_{m}\right\|_{0}\right)\right)\left\|p_{0}\right\|_{L^{1}}\right], \\
\leq & \frac{A}{\Gamma(\alpha)}\left[\left\|p_{0} p_{1}\right\|_{L^{1}}+W+\left(r\left(\left\|u_{m}\right\|_{0}\right)+r(1)+w\left(\left\|u_{m}\right\|_{0}\right)\right)\left\|p_{0}\right\|_{L^{1}}\right] .
\end{aligned}
$$

Also from Lemma 3.5, we obtain

$$
\left|D^{\delta} u_{m}(t)\right| \leq \frac{A}{\Gamma(\alpha-\delta)}\left[\left\|p_{0} p_{1}\right\|_{L^{1}}+W+\left(r\left(\left\|u_{m}\right\|_{0}\right)+r(1)+w\left(\left\|u_{m}\right\|_{0}\right)\right)\left\|p_{0}\right\|_{L^{1}}\right] .
$$

Consequently,

$$
\left\|u_{m}\right\|_{0} \leq \Upsilon\left[\left\|p_{0} p_{1}\right\|_{L^{1}}+W+\left(r\left(\left\|u_{m}\right\|_{0}\right)+r(1)+w\left(\left\|u_{m}\right\|_{0}\right)\right)\left\|p_{0}\right\|_{L^{1}}\right]
$$


Now from $\left(H_{3}\right)$, since $\lim _{\vartheta \rightarrow \infty} \frac{r(\vartheta)+w(\vartheta)}{\vartheta}=0$, then there exists an adequately large number $S>0$ such that

$$
\Upsilon\left[\left\|p_{0} p_{1}\right\|_{L^{1}}+W+(r(\vartheta)+r(1)+w(\vartheta))\left\|p_{0}\right\|_{L^{1}}\right]<\vartheta \text { for each } \vartheta \geq S,
$$

therefore,

$$
\left\|u_{m}\right\|_{0}<S \text { for } m \in N \text {. }
$$

Hence, the sequences $\left\{u_{m}\right\}$ and $\left\{D^{\delta} u_{m}\right\}$ are uniformly bounded in $C[0,1]$.

Now, we demonstrate that the sequences $\left\{u_{m}\right\}$ and $\left\{D^{\delta} u_{m}\right\}$ are equicontinuous in $C[0,1]$. As in Lemma 4.1 , let $0 \leq t_{1}<$ $t_{2} \leq 1$, then

$$
\begin{aligned}
\left|u_{m}\left(t_{2}\right)-u_{m}\left(t_{1}\right)\right|= & \left|\int_{0}^{1}\left(G\left(t_{2}, s\right)-G\left(t_{1}, s\right)\right) f_{m}\left(t, u_{m}(t), D^{\delta} u_{m}(t)\right)\right|, \\
= & \mid A\left(t_{2}^{\alpha-1}-t_{1}^{\alpha-1}\right)\left[\int_{0}^{1} \frac{(1-s)^{\alpha-1}}{\Gamma(\alpha)} f_{m}\left(s, u_{m}(s), D^{\delta} u_{m}(s)\right) d s\right. \\
& \left.-\lambda \int_{0}^{1} \frac{(1-s)^{\alpha+\gamma-1}}{\Gamma(\alpha+\gamma)} f_{m}\left(s, u_{m}(s), D^{\delta} u_{m}(s)\right) d s\right] \\
& +\int_{0}^{t_{1}} \frac{\left(t_{2}-s\right)^{\alpha-1}-\left(t_{1}-s\right)^{\alpha-1}}{\Gamma(\alpha)} f_{m}\left(s, u_{m}(s), D^{\delta} u_{m}(s)\right) d s \\
& +\int_{t_{1}}^{t_{2}} \frac{\left(t_{2}-s\right)^{\alpha-1}}{\Gamma(\alpha)} f_{m}\left(s, u_{m}(s), D^{\delta} u_{m}(s)\right) d s \mid .
\end{aligned}
$$

It follow from (4.10) and (4.11) that

$$
\begin{aligned}
\mid u_{m}\left(t_{2}\right)- & u_{m}\left(t_{1}\right) \mid \\
\leq & A\left|t_{2}^{\alpha-1}-t_{1}^{\alpha-1}\right|\left[\int_{0}^{1} \frac{(1-s)^{\alpha-1}}{\Gamma(\alpha)} p_{0}(s)\left(p_{1}(s)+q\left(\frac{\rho s(1-s)^{2}}{2 \Gamma(\alpha)}\right)+r(S)+r(1)+w(S)\right) d s\right. \\
& \left.+\lambda \int_{0}^{1} \frac{(1-s)^{\alpha+\gamma-1}}{\Gamma(\alpha+\gamma)} p_{0}(s)\left(p_{1}(s)+q\left(\frac{\rho s(1-s)^{2}}{2 \Gamma(\alpha)}\right)+r(S)+r(1)+w(S)\right) d s\right] \\
& +\int_{0}^{t_{1}} \frac{\left(t_{2}-s\right)^{\alpha-1}-\left(t_{1}-s\right)^{\alpha-1}}{\Gamma(\alpha)} p_{0}(s)\left(p_{1}(s)+q\left(\frac{\rho s(1-s)^{2}}{2 \Gamma(\alpha)}\right)+r(S)+r(1)+w(S)\right) d s \\
& +\frac{\left(t_{2}-t_{1}\right)^{\alpha-1}}{\Gamma(\alpha)} \int_{t_{1}}^{t_{2}} p_{0}(s)\left(p_{1}(s)+q\left(\frac{\rho s(1-s)^{2}}{2 \Gamma(\alpha)}\right)+r(S)+r(1)+w(S)\right) d s .
\end{aligned}
$$

Also we have

$$
\begin{aligned}
& \mid D^{\delta} u_{m}\left(t_{2}\right)-D^{\delta} u_{m}\left(t_{1}\right) \mid \\
& \leq \frac{A \Gamma(\alpha)\left|t_{2}^{\alpha-\delta-1}-t_{1}^{\alpha-\delta-1}\right|}{\Gamma(\alpha-\delta)}\left[\int_{0}^{1} \frac{(1-s)^{\alpha-1}}{\Gamma(\alpha)} f_{m}\left(t, u_{m}(t), D^{\delta} u_{m}(t)\right) d s\right. \\
&\left.+\lambda \int_{0}^{1} \frac{(1-s)^{\alpha+\gamma-1}}{\Gamma(\alpha+\gamma)} f_{m}\left(s, u_{m}(s), D^{\delta} u_{m}(s)\right) d s\right] \\
&+\int_{0}^{t_{1}} \frac{\left(t_{2}-s\right)^{\alpha-\delta-1}-\left(t_{1}-s\right)^{\alpha-\delta-1}}{\Gamma(\alpha-\delta)} f_{m}\left(s, u_{m}(s), D^{\delta} u_{m}(s)\right) d s, \\
&+\int_{t_{1}}^{t_{2}} \frac{\left(t_{2}-s\right)^{\alpha-\delta-1}}{\Gamma(\alpha-\delta)} f_{m}\left(s, u_{m}(s), D^{\delta} u_{m}(s)\right) d s \quad \text { if } \alpha-\delta>1, \\
&\left|D^{\delta} u_{m}\left(t_{2}\right)-D^{\delta} u_{m}\left(t_{1}\right)\right| \leq \int_{t_{1}}^{t_{2}} f_{m}\left(s, u_{m}(s), D^{\delta} u_{m}(s)\right) d s, \quad \text { if } \alpha-\delta=1 .
\end{aligned}
$$


Hence

$$
\begin{aligned}
\mid D^{\delta} u_{m}\left(t_{2}\right)- & D^{\delta} u_{m}\left(t_{1}\right) \mid \\
\leq & \frac{A \Gamma(\alpha)\left|t_{2}^{\alpha-\delta-1}-t_{1}^{\alpha-\delta-1}\right|}{\Gamma(\alpha-\delta)}\left[\int_{0}^{1} \frac{(1-s)^{\alpha-1}}{\Gamma(\alpha)} p_{0}(s)\right. \\
& \times\left(p_{1}(s)+q\left(\frac{\rho s(1-s)^{2}}{2 \Gamma(\alpha)}\right)+r(S)+r(1)+w(S)\right) d s \\
+ & \left.\lambda \int_{0}^{1} \frac{(1-s)^{\alpha+\gamma-1}}{\Gamma(\alpha+\gamma)} p_{0}(s)\left(p_{1}(s)+q\left(\frac{\rho s(1-s)^{2}}{2 \Gamma(\alpha)}\right)+r(S)+r(1)+w(S)\right) d s\right] \\
+ & \int_{0}^{t_{1}} \frac{\left(t_{2}-s\right)^{\alpha-\delta-1}-\left(t_{1}-s\right)^{\alpha-\delta-1}}{\Gamma(\alpha-\delta)} p_{0}(s) \\
& \times\left(p_{1}(s)+q\left(\frac{\rho s(1-s)^{2}}{2 \Gamma(\alpha)}\right)+r(S)+r(1)+w(S)\right) d s \\
+ & \frac{\left(t_{2}-t_{1}\right)^{\alpha-\delta-1}}{\Gamma(\alpha-\delta)} \int_{t_{1}}^{t_{2}} p_{0}(s)\left(p_{1}(s)+q\left(\frac{\rho s(1-s)^{2}}{2 \Gamma(\alpha)}\right)+r(S)+r(1)+w(S)\right) d s
\end{aligned}
$$

and

$$
\begin{gathered}
\left|D^{\delta} u_{m}\left(t_{2}\right)-D^{\delta} u_{m}\left(t_{1}\right)\right| \leq \int_{t_{1}}^{t_{2}} p_{0}(s)\left(p_{1}(s)+q\left(\frac{\rho s(1-s)^{2}}{2 \Gamma(\alpha)}\right)+r(S)+r(1)+w(S)\right) d s \\
\text { if } \alpha-\delta=1 .
\end{gathered}
$$

Now, choosing an arbitrary $\epsilon>0$, there exists a positive number $\delta(\epsilon)$ such that when $\left|t_{2}-t_{1}\right|<\delta$, then $\left|u_{m}\left(t_{2}\right)-u_{m}\left(t_{1}\right)\right|<\epsilon$ and $\left|D^{\delta} u_{m}\left(t_{2}\right)-D^{\delta} u_{m}\left(t_{1}\right)\right|<\epsilon$. Consequently, $\left\{u_{m}\right\}$ and $\left\{D^{\delta} u_{m}\right\}$ are equicontinuous in $C[0,1]$. Thus by Arzelà-Ascoli theorem $\left\{u_{m}\right\}$ and $\left\{D^{\delta} u_{m}\right\}$ are relatively compact in $\mathcal{E}$.

\section{Positive solutions of SFDE with FIBCs (1.1)-(1.2)}

Theorem 5.1. Let $\left(H_{1}\right)-\left(H_{4}\right)$ hold. Then SFDE (1.1) with FIBCs (1.2) has a multiple positive solution $u$ and

$$
u(t) \geq \frac{\rho t(1-t)^{2}}{2 \Gamma(\alpha)}, \quad t \in[0,1]
$$

Proof. Lemmas 4.2 and 4.3 guarantee that regular FDE (4.4) with FIBCs (1.2) has a positive solution $u_{m}, m \in N$ satisfying (4.9) and the sequences $\left\{u_{m}\right\}$ and $\left\{D^{\delta} u_{m}\right\}$ are relatively compact in $\mathcal{E}$. Thus there exist $u \in \mathcal{E}$ and a subsequence $\left\{u_{n_{m}}\right\}$ of $\left\{u_{m}\right\}$ such that $\lim _{m \rightarrow \infty} u_{n_{m}}=u$ in $\mathcal{E}$. Consequently, $u \in \mathcal{Q}, u$ fulfills (5.1) and

$$
\lim _{m \rightarrow \infty} f_{n_{m}}\left(t, u_{n_{m}}(t), D^{\delta} u_{n_{m}}(t)\right)=f\left(t, u(t), D^{\delta} u(t)\right), \text { a.e. } t \in[0,1] .
$$

For a.e. $s \in[0,1]$ and all $t \in[0,1], m \in N$, using inequalities (4.3), (4.9), (4.11) and Lemma 3.3(iii), we have

$$
0 \leq G(t, s) f_{m}\left(s, u_{m}(s), D^{\delta} u_{m}(s)\right) \leq \frac{A}{\Gamma(\alpha)} p_{0}(s)\left(p_{1}(s)+q\left(\frac{\rho s(1-s)^{2}}{2 \Gamma(\alpha)}\right)+r(S)+r(1)+w(S)\right)
$$

holds. Hence by the Lebesgue dominated convergence theorem

$$
\lim _{m \rightarrow \infty} \int_{0}^{1} G(t, s) f_{n_{m}}\left(s, u_{n_{m}}(s), D^{\delta} u_{n_{m}}(s)\right) d s=\int_{0}^{1} G(t, s) f\left(s, u(s), D^{\delta} u(s)\right) d s .
$$

Since

$$
u_{n_{m}}(t)=\int_{0}^{1} G(t, s) f_{n_{m}}\left(s, u_{n_{m}}(s), D^{\delta} u_{n_{m}}(s)\right) d s, \quad t \in[0,1]
$$


as $m \rightarrow \infty$ in the last equality, at that point we have

$$
u(t)=\int_{0}^{1} G(t, s) f\left(s, u(s), D^{\delta} u(s)\right) d s, \quad t \in[0,1] .
$$

Consequently, $u$ is a positive solution of SFDE (1.1) with FIBCs (1.2).

\section{References}

[1] B. Ahmad and S.K. Ntouyas, A note on fractional differential equations with fractional separated boundary conditions, Abstract and Applied Analysis, vol. 2012, (2012) 11 pages, Article ID 818703.

[2] Z. Bai, On positive solutions of a nonlocal fractional boundary value problem, Nonlinear Analysis: Theory, Methods and Applications, 72( 2) (2010) ) 916-924.

[3] D. Baleanu, K. Diethelm, E. Scalas, and J.J. Trujillo, Fractional Calculus Models and Numerical Methods. Series on Complexity, Nonlinearity and Chaos, vol. 3, World Scientific, Boston, Mass, USA, 2012.

[4] A. Cabada and G. Wang, Positive solutions of nonlinear fractional differential equations with integral boundary value conditions. J. Math. Anal. Appl. 389 (2012), 403-411.

[5] A.M.A. El-Sayed and Sh.A. Abd El-Salam, Nonlocal boundary value problem of a fractional-order functional differential equation, Int. J. Nonlinear Science, 7 (4) (2009) 436-442.

[6] A.M.A. El-Sayed and E.O. Bin-Taher, Positive solutions for a nonlinear multi-point boundary-value problem of fractional and second order, Electronic J. Diff. Equ., 2013(64) 2013, 1-8.

[7] J. Sabatier, O.P. Agrawal and J.A.T. Machado, Eds., Advances in fractional calculus: Theoretical developments and applications in physics and engineering, Springer, Dordrecht, The Netherlands, 2007.

[8] Z. Wei, C. Pang and Y. Ding, Positive solutions of singular Caputo fractional differential equations with integral boundary conditions. Commun. Nonlinear Sci. Numer. Simul. 17 (2012) 3148-3160.

[9] J. Zhao, P. Wang and W. Ge, Existence and nonexistence of positive solutions for a class of third order BVP with integral boundary conditions in Banach spaces. Commun. Nonlinear Sci. Numer. Simul. 16 (2011) $402-413$.

[10] S. Taliaferro, A nonlinear singular boundary value problem, Nonlinear Anal. 3 (1979) 897-904.

[11] R. Aris, The Mathematical Theory of Diffusion and Reaction of Permeable Catalysts Clarendon, Oxford (1975).

[12] A. Callegari and A. Nachman, A nonlinear singular boundary value problem in the theory of pseudoplastic fluids, SIAM J. Appl. Math. 38 (1980) 275-282.

[13] T. Qui and Z. Bai, Existence of positive solutions for singular fractional differential equations, Electron J. Differential Equations 2008 (146) (2008) 1-9.

[14] Z. Bai and T. Qui, Existence of positive solution for singular fractional differential equation, Appl. Math. Comput. 215 (2009) 2761-2767.

[15] Y. Tian and A. Chen, The existence of positive solution to three-point singular boundary value problem of fractional differential equation, Abstr. Appl. Anal. 2009 (2009). Article ID 314656, doi:10.1155/2009/314656.

[16] R.P. Agarwal, D. O'Regan and S. Staněk, Positive solutions for Dirichlet problems of singular nonlinear fractional differential equations, J. Math. Anal. Appl. 371 (2010) 57-68.

[17] Z. Bai and W. Sun, Existence and multiplicity of positive solutions for singular fractional boundary value problems, Comput. Math. Appl. 63 (2012) 1369-1381.

[18] J.W. Lyons and J.T. Neugebauer, Positive solutions of a singular fractional boundary value problem with a fractional boundary condition, Opuscula Math. 37(3) (2017) 421-434. 
[19] Y. Qiao and Z. Zhou, Existence of positive solutions of singular fractional differential equations with infinite-point boundary conditions, Adv. Differ. Equ., (2017) 2017:8, DOI 10.1186/s13662-016-1042-9.

[20] S. Staněk, The existence of positive solutions of singular fractional boundary value problems, Comput. Math. Appl. 62 (2011) 1379-1388.

[21] X. Xu, D. Jiang and C. Yuan, Multiple positive solutions for the boundary value problem of a nonlinear fractional differential equation, Nonlinear Anal. 71 (2009) 4676-4688.

[22] C. Yuan, D. Jiang and X. Xu, Singular positone and semipositone boundary value problems of nonlinear fractional differential equations, Math. Probl. Eng. 2009 (2009). Article ID 533207, 17 pages, doi:10.1155/2009/535209.

[23] A. Cabada and Z. Hamdi, Nonlinear fractional differential equations with integral boundary value conditions, Appl. Math. Comput. 228 (2014) 251-257.

[24] H. Li, L. Liu and Y. Wu, Positive solutions for singular nonlinear fractional differential equation with integral boundary conditions. Bound. Value Probl. 2015, 232 (2015).

[25] J.A. Nanware and D.B. Dhaigude, Existence and uniqueness of solutions of differential equations of fractional order with integral boundary conditions. J. Nonlinear Sci. Appl. 7 (2014) 246-254.

[26] Y. Sun and M. Zhao, Positive solutions for a class of fractional differential equations with integral boundary conditions, Appl. Math. Lett. 34 (2014) 17-21.

[27] X. Zhang, L. Wang and Q. Sun, Existence of positive solutions for a class of nonlinear fractional differential equations with integral boundary conditions and a parameter. Appl. Math. Comput. 226 (2014) 708-718.

[28] T. Wang and F. Xie, Existence and uniqueness of fractional differential equations with integral boundary conditions. J. Nonlinear Sci. Appl. 1(4) (2008) 206-212.

[29] A.A. Kilbas, H.M. Srivastava and J.J. Trujillo, Theory and applications of fractional differential equations, NorthHolland Mathematics Studies 204, Editor: Jan van Mill, Elsevier, Amsterdam, The Netherlands 2006.

[30] M.A. Krasnosel'skii, Positive Solutions of Operator Equations, P. Noordhoff, Groningen, The Netherlands, 1964.

[31] X. Su, Boundary value problem for a coupled system of nonlinear fractional differential equations, Applied Mathematics Letters, 22(1) (2009) 64-69. 